\title{
Changes in walking levels of people with stroke following discharge from hospital: A pilot study
}

Emily Kate Timothy, MHealsc(Rehabilitation), BSc(Hons) Physiotherapy

Physiotherapist, Canterbury District Health Board, New Zealand

Braidie-Jean Bown, BPhty

Physiotherapist, Canterbury District Health Board, New Zealand

Rachael M Beever, BPhty

Physiotherapist, Canterbury District Health Board, New Zealand

Hilda F Mulligan, PhD

Senior Lecturer, Centre for Health, Activity and Rehabilitation Research, School of Physiotherapy, University of Otago, New Zealand

\section{ABSTRACT}

This study aimed to investigate changes in walking levels of independently mobile individuals following a stroke between the inpatient setting and the home environment, both directly after discharge and in the longer term. Forty-three participants who had a stroke as their primary diagnosis and who could walk 10 metres without the support of another person on discharge from hospital completed the study. The Step-Watch Activity Monitor, six minute walk test, 10 metre walk test, and the Stroke Impact Scale were used to measure outcome. There was a significant increase in number of steps taken per day between 3-14 days and 4-6 months post-discharge $(p=0.0001)$. Walking speed, six minute walk test, perceived mobility and perceived ability to perform activities of daily living all had strong positive correlations with average amount of steps at 4-6 months post-discharge from hospital and could therefore be used as predictors of walking level in the longer term. However, step counts are still below those required for health benefits. This suggests it is beneficial for further emphasis to be placed on increasing activity levels for people after stroke, even if they are able to mobilise independently on discharge home.

\section{Timothy, E., Bown, B., Beever, R., \& Mulligan, H. (2017). Changes in walking levels of people with stroke following discharge from hospital: A pilot study. New Zealand Journal of Physiotherapy 45(1): 17-23. doi: 10.15619/NZJP/45.1.03}

Key words: Stroke, Step count, Walking, Community.

$\ldots \ldots \ldots \ldots \ldots \ldots \ldots \ldots \ldots \ldots \ldots \ldots \ldots \ldots \ldots \ldots \ldots \ldots \ldots \ldots \ldots \ldots \ldots \ldots \ldots \ldots \ldots \ldots \ldots \ldots \ldots \ldots \ldots \ldots \ldots \ldots \ldots \ldots \ldots \ldots \ldots$

\section{INTRODUCTION}

Stroke affects approximately 6000 New Zealanders per year (Tobias, Cheung, Carter, Anderson \& Feigin, 2007). Although the incidence of stroke is increasing due to an ageing population, mortality is decreasing (Ministry of Health, 2014; Tobias et al., 2007). This means that an increasing number of people are living with the effects of a stroke. Stroke has a physical, psychological and financial impact on both individuals and their communities (Gbiri, Olawale \& Isaac, 2015). If current trends continue, there will be 70 million people living with stroke globally by 2030 (Feigin et al., 2014), thus increasing the burden on health care systems and communities. Stroke can reduce a person's quality of life by limiting their ability to participate in home and community life (Carod-Artal, Egido, González \& Seijas, 2000; Chen \& Rimmer, 2011). Therefore an important goal after stroke is to support people to reintegrate into their community. A factor in achieving this is for people after stroke to have adequate levels of physical activity including the ability to walk in their community (Van Peppen et al., 2004).

It is well documented that activity levels of people after stroke are low not only during inpatient rehabilitation but also after reintegration into the community (Bernhardt, Dewey, Thrift \& Donnan 2004; Skarin et al., 2014; West \& Bernhardt, 2012). In the inpatient setting, studies have found that patients spend most of their time inactive, alone or in their rooms (Bernhardt et al., 2004; Skarin et al., 2014; West \& Bernhardt, 2012). Even after discharge back to community life, people have reduced levels of physical activity after stroke (Field, Gebruers, Sundaram, Nicholson \& Mead, 2013; Rand, Eng, Tang, Jeng \& Hung 2009; Tudor-Locke et al., 2011). For instance, a recent meta-analysis of studies in 14 different countries (including New Zealand and Australia) showed that people after stroke take an average of 4355.2 steps per day (Field et al., 2013). This is well below the recommended 7000 steps for the older adult population (TudorLocke et al., 2011) and mirrors evidence that people participate in $36 \%$ less activity after stroke than normative values for community dwelling individuals over the age of 60 (Rand et al., 2009).

Decreased levels of physical activity during hospitalisation result in deconditioning (Billinger et al., 2014), which may contribute to low activity levels at home despite the greater functional demands of a home environment compared to a hospital environment. Although we know that activity levels of people after stroke remain below the recommended levels for maintaining health and quality of life (Billinger et al., 2014; Field et al., 2013), we do not yet have sufficient information about changes in activity levels from discharge to home living, in the shorter and then the longer term. A clear description of changes in activity levels over time will guide rehabilitation 
goal setting and help create effective and efficient interventions after stroke. The main aim of this study was to investigate changes in walking levels of independently mobile individuals following a stroke between the inpatient setting and the home environment, both directly after discharge and in the longer term.

\section{METHODS}

This was a longitudinal observational study which took place between 2013 and 2015 in a large district health board in a metropolitan area of New Zealand. Ethical approval for this study was granted by the University of Otago Human Ethics Committee (13/002).

\section{Recruitment and participants}

Hospitalised patients with stroke were recruited from a stroke rehabilitation unit and an acute stroke unit. Individuals were eligible to participate in this study if they were hospitalised with a stroke as their primary diagnosis, could walk 10 metres independently prior to discharge with or without an aid and could apply (or have support to apply) an activity monitor around their ankle for waking hours over three days. Individuals were excluded if they could not walk 10 metres prior to the stroke, were living in a private care facility, were medically unwell or their cognition precluded them from providing informed consent.

Physiotherapists based in the stroke rehabilitation unit and acute stroke unit, and who were not otherwise involved in the study, screened all patients against a recruitment checklist and provided eligible people with an information sheet. People who were interested in participating were met by a member of the research team (BB, RB, ET, also physiotherapists, but not working on these wards), to explain the study further. People provided written informed consent to participate in the study.

\section{Procedures}

Demographic data regarding each participant's age, sex and classification/location of stroke were collected at the time of recruitment from patient notes. Ethnicity was collected by self-report. Further information including walking aids, living situation (alone or with others) and an estimation of house size (small, medium or large) was collected by the research team at the time of recruitment.

Primary and secondary outcome measures were completed by the participant's treating physiotherapist during their inpatient stay for participants recruited from the stroke rehabilitation unit (Assessment A) and by community stroke physiotherapists on two home visits after discharge from hospital (Assessments $B$ and C). The first community visit was between 3-14 days after discharge and the second occurred at 4-6 months.

The primary outcome measure used was the Step-Watch Activity Monitor (SAM). The SAM consists of an accelerometer and electronic filter that detects leg movement to determine the amount of steps taken by its wearer. It is worn above the lateral malleolus on either leg. Information from the SAM was downloaded through a docking system to a computer, with no information displayed on the SAM. Therefore, participants were unable to see how many steps they took. The SAM is a valid and reliable measure of steps taken per day in stroke (Mudge \&
Stott, 2009; Storti et al., 2008). Participants were asked to wear the SAM from waking to going to bed for three days.

The six minute walk test (6MWT), Stroke Impact Scale (SIS) and the 10 metre walk test (10MWT) were used as secondary outcome measures. The 10MWT and 6MWT have been demonstrated as valid and reliable measures of walking ability after stroke (Enright et al., 2003; Flansbjer, Holmbäck, Downham \& Lexell, 2005; Kosak \& Smith, 2005; Wevers, Kwakkel \& van de Port, 2011; Wolf et al., 1999). These measures were selected to provide information on walking speed and endurance. For both outcome measures, assistive devices or orthotics could be used but were recorded and kept consistent from Assessment A through to C. The SIS is a selfrated questionnaire which indicates how stroke has subjectively impacted health and quality of life for the participant. It is a stroke specific, self-reported measure that contains eight domains: strength, hand function, activities of daily living (ADL), mobility, communication, emotion, memory and thinking, and participation, and has been demonstrated to be reliable, valid and sensitive to change (Duncan et al., 1999).

Participants came from either a stroke rehabilitation unit or an acute stroke unit. Those from the stroke rehabilitation unit had three sets of data collected; once in hospital (Assessment A) and twice in the community (Assessments B and C). Participants from the acute stroke unit only had two sets of community data collected (Assessments B and C). No inpatient data could be collected due to insufficient time between consenting to be in the study and being discharged home. Community data were collected at the participant's home, with the 6MWT carried out on the pavement outside the home or in the participant's driveway. This was consistent for Assessments B and C.

\section{Analysis}

The distribution of data was examined for normality using the Kolmogorov-Smirnov test (Antonius, 2012). Data were also assessed for homoscedascity and linearity by graphing the data. Wilcoxon signed rank tests with Bonferroni correction were used to examine the difference between steps over the three time periods. A Pearson's correlation analysis was performed to explore the relationship between different components of the SIS or walking speed with average number of steps (Antonius, 2012). Linear regression models were fitted to account for the impact of age on step counts with any predictive measures.

\section{RESULTS}

Sixty-four people were identified by the recruitment team for the study from the beginning of 2013 to July 2015. Fifty-one participants consented to participate in the study, but six people withdrew from the study and there were two incomplete data sets. Therefore a total of 43 people completed the study. See Figure 1 for the flow of participants through the study. The mean age of these participants was 75.8 years (SD 7.5). The group characteristics are presented in Table 1. All data were normally distributed except walking speed and mean steps at 3-14 days post-discharge. Assumptions of linearity and homoscedasticity were met with all data. Main results are shown in Table 2. 


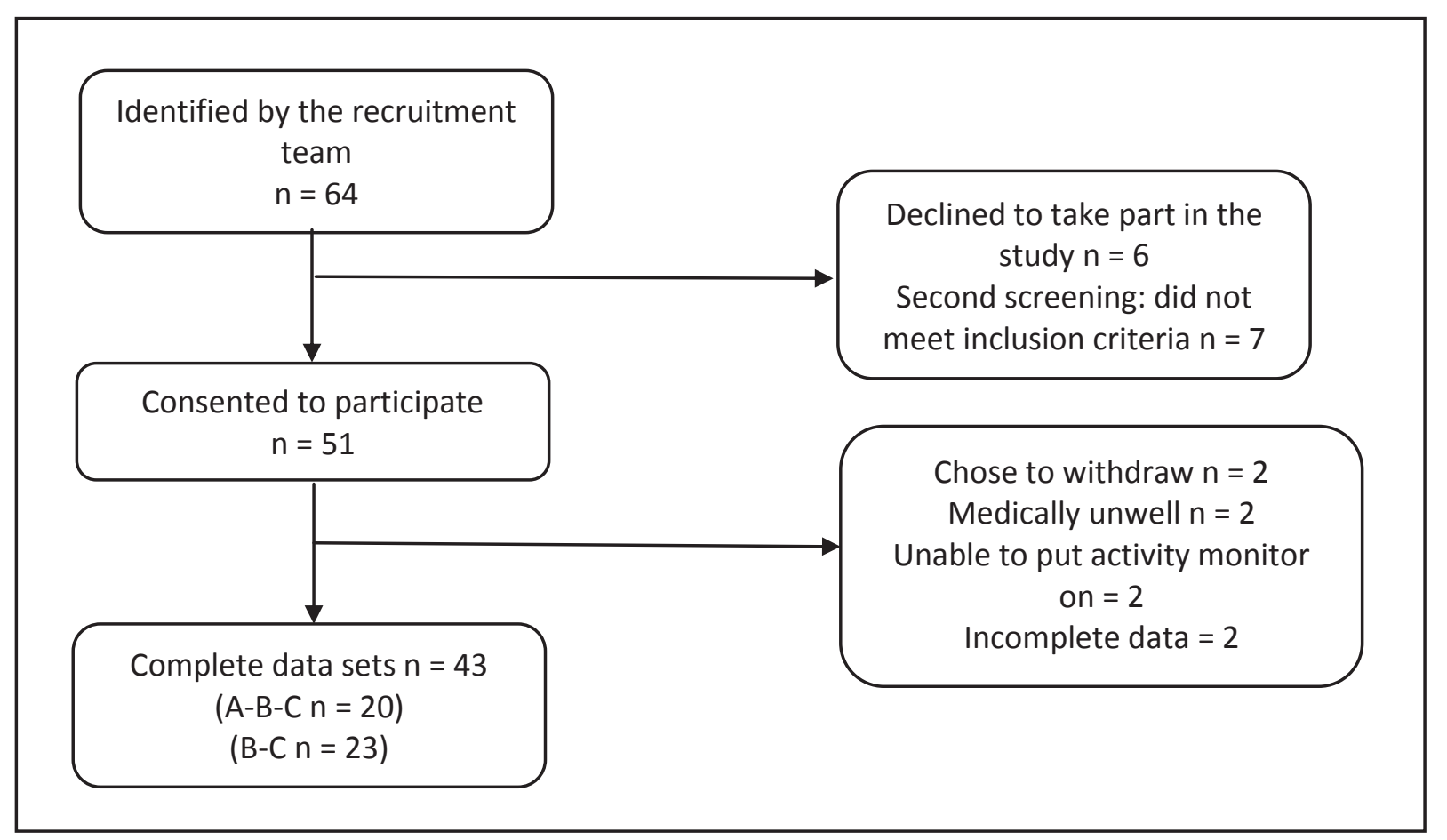

Figure 1. Flow chart outlining study recruitment, dropout and completion.

Table 1: Group characteristics

\begin{tabular}{lr}
\hline & $\mathbf{n}(\%)$ \\
\hline SEX & $25(58.1)$ \\
Female & $18(41.9)$ \\
\hline ETHNICITY & $39(90.7)$ \\
NZ European & $1(2.3)$ \\
Māori & $2(4.7)$ \\
Other European & $1(2.3)$ \\
Other (Russian) & \\
\hline CLASSIFICATION OF STROKE & $15(34.9)$ \\
PACI & $11(25.6)$ \\
LACI & $11(25.6)$ \\
POCI & $2(4.7)$ \\
ICH & $1(2.3)$ \\
Multiple infarcts & \\
\hline SIDE OF STROKE & $18(41.8)$ \\
Left & $21(48.8)$ \\
Right & $4(9.3)$ \\
Bilateral & $9(20)$ \\
\hline LIVING ALONE &
\end{tabular}

Notes: PACI, Partial anterior circulatory infarct; LACI, lacunar circulatory infarct; $\mathrm{POCl}$, posterior circulatory infarct; ICH, inter-cerebral haemorrhage.
A Wilcoxon Signed Rank Test revealed a significant increase in number of steps taken (as measured by SAM) between 3-14 days post discharge and 4-6 months post discharge with a medium effect size, $(p=0.0001)$. The mean number of steps increased from 2839.5 (SD 1324.9) at 3-14 days to 3665.7 steps (SD 1787.8) at 4-6 months post-discharge. There was also a significant change between inpatient data and 3-14 days post discharge ( $p=0.011$ ) as well as inpatient data and 4-6 months post discharge $(p=0.0001)$, although only 20 participants had been measured for the initial data set (Assessment $A$ ).

In addition to step count, walking speed over 10 metres and distance walked in six minutes were also measured. Both 10MWT and 6MWT were found to have a large positive correlation with steps per day at 4-6 months post-discharge $(r=0.59, p<0.0001 ; r=0.68, p<0.0001$, respectively). Four separate linear regression models were fitted to see if there was an impact of age on the step counts at 4-6 months postdischarge. Results were, however, consistent at all ages. All secondary outcome measures still had an individual significant effect after adjusting for age.

Additionally, relationships between mean number of steps 4-6 months post discharge, and components of the SIS, were investigated using the Pearson correlation coefficient. There was a strong positive correlation between perceived mobility and mean number of steps 4- 6 months post-discharge, $(r=0.60$, $p=<0.0001)$, with greater perceived mobility being associated with greater number of steps. Similarly, a large correlation $(r=0.57, p<0.0001)$ was also found with perceived ability to perform activities of daily living and number of steps 4-6 months post discharge. No significant correlations were found between any other relevant categories of the SIS (recovery, mood, strength and community participation) and number of steps 4-6 months post-discharge. 
Table 2: Objective outcome measures compared over three time periods

\begin{tabular}{llll}
\hline Outcome Measure & Assessment A & Assessment B & Assessment C \\
\hline Steps count, M (SD) & $1996.6(820.3)$ & $2839.5(1324.9)$ & $3665.7(1787.8)$ \\
\hline 6MWT distance m, M (SD) & $233.7(86.2)$ & $336.6(150.5)$ & $359.7(124.5)$ \\
\hline 10MWT speed m/s, M (SD) & $0.80(0.25)$ & $1.19(0.53)$ & $1.4(0.65)$ \\
\hline
\end{tabular}

Notes: 6MWT, Six minute walk test; 10MWT, Ten metre walk test; M, Mean; SD, standard deviation.

Ten metre walk test speed and 6MWT distance at 3-14 days post-discharge were assessed as predictors for mean amount of steps taken 4-6 months post-discharge. There was found to be a positive correlation, $(r=0.49, p=0.001$ and $r=0.36, p=0.019$ respectively) for both variables. A faster $10 \mathrm{MWT}$ speed and larger 6MWT distance at 3-14 days post-discharge were found to be associated with a greater amount of steps 4-6 months post-discharge. Furthermore, both the perceived mobility and ADL ability components of the SIS at 3-14 days post-discharge had significant positive correlations with amount of steps 4-6 months post-discharge; $r=0.49, p=0.001$ and $r=0.58, p<0.0001$ respectively. Thus larger scores in perceived mobility and ADL at 3-14 days post-discharge were associated with a greater amount of steps four months post-discharge. This indicates that all these variables could be used as predictors of daily step count for people post-discharge after stroke.

\section{DISCUSSION}

The primary aim of this study was to investigate changes in walking levels of independently mobile individuals following a stroke between the inpatient setting and the home environment, both directly after discharge and in the longer term. Our results showed a significant increase in the number of steps per day between inpatient stay, 3-14 days post discharge and 4-6 months post discharge. In addition, there was a strong positive correlation between 10MWT speed, 6MWT, SIS mobility and SIS ADL scores with steps per day at 4-6 months post discharge. The study also demonstrated that 10MWT speed, 6MWT, SIS mobility and SIS ADL scores directly after discharge could be used as predictors of walking levels in the longer term. Faster 10MWT speeds, increased 6MWT distance, higher SIS mobility and ADL scores at 3-14 days post discharge were correlated with a greater amount of steps 4-6 months post discharge. There was no correlation between other components of the SIS and daily step count, suggesting that SIS domains other than mobility and ADL cannot be used as predictors for walking levels.

Our study supports that of Manns and Baldwin (2009) who conducted a longitudinal observational study investigating differences in step count pre- and post-discharge in a stroke population. Both ours and the study by Manns and Baldwin (2009) had a similar increase in step count, of approximately 800 steps. Although Manns and Baldwin (2009) had a higher initial (5411) and final (6195) step count than in our study, Manns and Baldwin had a younger mean population age by approximately eight years. Similarly Shaughnessy, Michael, Sorkin and Macko (2005) and Moore et al. (2013) investigated walking activity of people after stroke using step count prior to discharge and found that people took 1536 (Shaughnessy et al., 2005) and 3111 (Moore et al., 2013) steps per day. This correlates with our participants who walked an average of 1997 steps per day as inpatients, again illustrating low inpatient activity within the stroke population (Field et al, 2013; Rand et al., 2009; Tudor-Locke et al, 2011). Participants' walking speeds according to the 6MWT and 10MWT were comparable to previously identified speeds in people with chronic stroke of similar age to those in our study (Flandbjer et al., 2005). However comparing our participants' walking speed with established norms for community dwelling older adults, we see that our participants' speeds were below the established norms even at 4-6 months post-discharge from hospital. For instance Steffan, Hacker and Mollinger (2002) found an average 6MWT distance of $499 \mathrm{~m}$ and a fast $10 \mathrm{MWT}$ speed of $1.77 \mathrm{~m} / \mathrm{s}$ for the 70-79 age group. While the mean age of our participants is within this age range, our 4-6 month 6MWT and 10MWT results were below these norms (360m and $1.4 \mathrm{~m} / \mathrm{s}$ ), meaning that our participants with stroke did not reach the expected levels and speed of walking as age matched peers. This may be in part due to participants in our study still being in a sub-acute phase of recovery. However, despite slow walking speeds amongst our participants, the mean walking speed for our participants when inpatients was faster than the suggested speed of $0.66 \mathrm{~m} / \mathrm{s}$ needed to achieve community ambulation (van de Port, Kwakkel \& Lindeman, 2008).

Our study has helped to establish how walking activity for people after stroke changes in the early stages following discharge from the inpatient setting. Such knowledge can be used to help guide goal setting in stroke rehabilitation by establishing norms for early post discharge activity levels. Community living demands the ability to mobilise safely and efficiently around the home and into the community, which requires adequate endurance and speed. The increase in 10MWT speed and 6MWT distance found in our study on return home may be explained by the requirement to adapt to these more demanding environments. However, at initial discharge people are likely to have a degree of deconditioning due to reduced activity over the inpatient stay (Bernhardt et al., 2014; West \& Bernhardt, 2012). Deconditioning could impair people's ability to undertake functional activities on return home after stroke, limiting their speed of recovery and leading to lower activity levels. Therefore, encouraging people with stroke to be more active in the inpatient environment could lead to a higher number of steps per day at discharge. A higher step count at discharge would lead to a higher step count on return home, and a further increase at 4-6 months post discharge. In particular, targeting people who have low walking speeds 
after stroke in the inpatient setting or on returning to the community, may better guide rehabilitation effort. However, previous studies have suggested that good outcomes on these standardised walking measures do not necessarily indicate people have resumed community ambulation (Lord, McPherson, McNaughton, Rochester \& Weatherall, 2004). Instead selfreported measures have been suggested as more useful indicators for community ambulation (Lord \& Rochester, 2005). Given that perceived walking ability and ADLs according to the SIS were strong indicators for step count at 4-6 months postdischarge, it may be that better utilisation of these measures by clinicians could enable more targeted rehabilitation interventions for walking activity. Further research is required to establish specific data ranges for these outcome measures that alert potential 'at risk' people for low walking activity in the longer term after stroke.

People with neurological conditions, including stroke, have been found to need more activity than their unaffected age related peers in order to gain health benefits and prevent secondary comorbidities (Billinger et al., 2014; Gallanagh, Quinn, Alexander \& Walters, 2011). Our study showed activity levels of participants were too low to gain health benefits, potentially leading to a cycle of increased comorbidities, further decreased activity and potential for re-hospitalisation. An ageing population and decreasing mortality from stroke will further increase the number of people with stroke potentially entering this cycle, which has the potential to exponentially increase the socioeconomic burden of stroke on the health care system. Therefore, it is imperative that we place more emphasis on increasing physical activity throughout the entire rehabilitation process. For example, in the hospital setting we could increase stimulation for walking activity by offering group activities for motivation (Eng et al., 2003) and encouraging people to be more engaged in rehabilitation activity, such as walking, outside of treatment sessions. In the community, easily accessible stroke specific exercise classes would not only improve activity levels but empower people after stroke and encourage support within a local stroke population. Pedometers or pedometer-like devices could also motivate people after stroke to increase their activity levels as they can independently monitor the progress towards their goals (Tudor-Locke, 2002).

Our study had some limitations. First, this study had a small sample size, particularly relating to inpatient data. This could be due to the strict inclusion criteria coupled with a busy hospital environment which meant not all possible participants were screened and invited into the study. Additionally only a specific cohort, i.e. those who were more physically able, was investigated. Furthermore our data were collected, both on the ward and in the community, by physiotherapists who may have also been a participant's treating physiotherapist. This means there was potential for bias both in terms of assessment by the person collecting the data, and performance of participants who may have wished to 'please' their physiotherapist, thus instituting a potential Hawthorne effect (Jones, 1992). However, the outcome measures chosen for this study have high inter-reliability and validity and are well supported in the literature for use in the stroke population (Duncan et al., 1999; Enright et al., 2003; Flansbjer et al., 2005; Kosak \&
Smith, 2005; Wevers et al., 2011; Wolf et al., 1999). SAMs are the most reliable movement sensors at low gait speeds and are equal to other pedometers at medium and high gait speeds (Mudge \& Stott, 2009; Storti et al., 2008). The SAM also blinded the wearer and assessor to its readings with results being uploaded electronically. Good reliability has been demonstrated for the SAM when worn for three consecutive days (Mudge \& Stott, 2008), suggesting that the three days of SAM data would give a valid measure of walking levels. However, the accuracy of SAM relies on the apparatus being applied for all waking hours, thus it is possible steps were not recorded if the SAM was not applied for all waking hours.

This study supports contemporary literature about activity levels of people after stroke in reporting that levels are lower than those required for health benefits. Future research could build on our study by exploring rehabilitation interventions to target ways to increase walking activity especially amongst those people after stroke who can be identified as being of 'high risk' according to predictive outcome measures.

\section{CONCLUSION}

Participants increased their number of steps taken per day over the months following discharge from hospital. This increase was predicted by walking speed and perceived function directly after discharge. However step counts are still below those required for health benefits. This suggests it is beneficial for further emphasis to be placed on increasing activity levels for people after stroke, even if they are able to mobilise independently on discharge home.

\section{KEY POINTS}

1. After stroke people increased the number of steps they took per day between the inpatient setting and the months after discharge from hospital, but they are still below those required for health benefits.

2. 10MWT speed, 6MWT, SIS mobility and SIS ADL at 3-14 days post-discharge were predictors of the number of steps taken at 4-6 months. This knowledge can help to identify people at risk of low activity.

3. Further emphasis should be placed on increasing activity levels over the entire rehabilitation process to reduce the future burden of stroke.

\section{PERIMISSIONS}

Ethical approval for this study was granted by the University of Otago Human Ethics Committee (13/002).

\section{DISCLOSURES}

The authors declare that there is no conflict of interest. Funding was provided by the Canterbury District Health Board and Canterbury Health Care of the Elderly Trust.

\section{ACKNOWLEDGEMENTS}

We would also like to thank Ma Yi biostatistician, and the University of Otago physiotherapy students Kara Fowke, Hannah Malloch, Brendon Moore, Kirsty Prattley, and Daniel Wong for their valuable contribution to this project, as well as those from 
the wider clinical team who assisted with recruitment and data collection.

\section{ADDRESS FOR CORRESPONDENCE}

Mrs Emily Timothy, Canterbury District Health Board, Community Stroke Rehabilitation Service, PO Box 1600, Christchurch 8140, New Zealand. Telephone: +64 33640640. Email: Emily.k.timothy@gmail.com.

\section{REFERENCES}

Antonius, R. (2012). Interpreting Quantitative Data with IBM SPSS Statistics $\left(2^{\text {nd }} \mathrm{Ed}\right)$. London: Sage.

Bernhardt, J., Dewey, H., Thrift, A., \& Donnan, G. (2004). Inactive and alone: physical activity within the first 14 days of acute stroke unit care. Stroke 35(4),1005-9. doi: 10.1161/01.STR.0000120727.40792.40.

Billinger, S.A., Arena, R., Bernhardt, J., Eng, J.J., Franklin, B.A., Johnson, C.M., et al (2014). Physical activity and exercise recommendations for stroke survivors: a statement for healthcare professionals from the American Heart Association/American Stroke Association. Stroke, 45(8), 2532-53. doi: 10.1161/STR.0000000000000022.

Carod-Artal, J., Egido, J.A., González, J.L., \& Seijas, V. (2000). Quality of life among stroke survivors evaluated 1 year after stroke: Experience of a stroke unit. Stroke, 31(12), 2995-3000. doi: 10.1161/01.STR.31.12.2995.

Chen, M.D., \& Rimmer, J.H. (2011). Effects of exercise on quality of life in stroke survivors: a meta-analysis. Stroke, 42(3), 832-7. doi: 10.1161/ STROKEAHA.110.607747.

Duncan, P.W., Wallace, D., Lai, S.M., Johnson, D., Embretson, S., \& Laster, L.J. (1999). The stroke impact scale version 2.0: evaluation of reliability, validity, and sensitivity to change. Stroke, 30(10), 2131-40. doi: 10.1161/01.STR.30.10.2131.

Eng, J.J., Chu, K.S., Kim, C.M., Dawson, A.S., Carswell, A., \& Hepburn, K.E. (2003). A community-based group exercise program for persons with chronic stroke. Medicine and Science in Sports and Exercise, 35(8), 1271 8. doi:10.1249/01.MSS.0000079079.58477.0B.

Enright, P.L., McBurnie, M.A., Bittner, V., Tracy, R.P., McNamara, R., Arnold, A., et al. (2003). The 6-min walk test: a quick measure of functional status in elderly adults. Chest, 123(2), 387-98. doi:10.1378/chest.123.2.387.

Feigin, V.L., Forouzanfar, M.H., Krishnamurthi, R., Mensah, G.A., Connor, M., \& Bennet, D.A. (2014). Global and regional burden of stroke during 19902010: Findings from the Global Burden of Disease Study 2010. Lancet, 383(9913), 245-55. doi:10.1016/S0140-6736(13)61953-4.

Field, M.J., Gebruers, N., Sundaram, T.S., Nicholson, S., \& Mead, G. (2013). Physical activity after stroke: A systematic review and meta-analysis. ISRN Stroke, 13. Doi: 10.1155/2013/464176.

Flansbjer, U.B., Holmbäck, A.M., Downham, D., \& Lexell, J. (2005). Reliability of gait performance tests in men and women with hemiparesis after stroke. Journal of Rehabilitation Medicine, 37(2), 75-82. doi: 10.1080/16501970410017215.

Gallanagh, S., Quinn, T.J., Alexander, J., \& Walters, M. (2011). Physical activity in the prevention and treatment of stroke. ISRN Neurology 2011. doi:10.5402/2011/953818.

Gbiri, C.A., Olawale, O.A., \& Isaac, S.O. (2015). Stroke management: Informal caregivers' burdens and strains of caring for stroke survivors. Annals of Physical and Rehabilitation Medicine, 58(2), 98-103. doi:10.1016/j.rehab.2014.09.017

Jones, S.R.G. (1992). Was There a Hawthorne Effect? American Journal of Sociology, 98(3), 451-68.
Kosak, M.S., \& Smith, T. (2005). Comparison of the 2-, 6-, and 12-minute walk tests in patients with stroke. Journal of Rehabilitation Research and Development, 42, 103-7.

Lord, S.E., \& Rochester, L. (2005). Measurement of community ambulation after stroke: Current status and future developments. Stroke, 36(7), 145761. doi: 10.1161/01.STR.0000170698.20376.2e.

Lord, S.E., McPherson, K., McNaughton, H.K., Rochester, L. \& Weatherall, M. (2004). Community ambulation after stroke: how important and obtainable is it and what measures appear predictive? Archives of Physical Medicine and Rehabilitation, 85(2), 234-9. doi:10.1016/j. apmr.2003.05.002.

Manns, P.J., \& Baldwin, E. (2009). Ambulatory activity of stroke survivors. Stroke, 40(3), 864-7. doi: 10.1161/STROKEAHA.108.531590.

Ministry of Health. Mortality and Demographic Data (2011). Wellington: Ministry of Health; 2014. p. 48-53.

Moore, S.A., Hallsworth, K., Plötz, T., Ford, G.A., Rochester, L., \& Trenell, M. (2013). Physical activity, sedentary behaviour and metabolic control following stroke: a cross-sectional and longitudinal study. PLOS ONE, 8(1), e55263. doi: 10.1371/journal.pone.0055263.

Mudge, S., \& Stott, N.S. (2008). Test-retest reliability of the Step Watch Activity Monitor outputs in individuals with chronic stroke. Clinical Rehabilitation, 22(10-11), 871-7. doi: 10.1177/0269215508092822.

Mudge, S., \& Stott, N.S. (2009). Timed walking tests correlate with daily step activity in persons with stroke. Archives of Physical Medicine and Rehabilitation, 90(2), 296-301. doi:10.1016/j.apmr.2008.07.025.

Rand, D., Eng, J.J., Tang, P.F., Jeng, J., \& Hung, C. (2009). How active are people with stroke? Use of accelerometers to assess physical activity. Stroke, 40(1), 163-8. doi: 10.1161/STROKEAHA.108.523621.

Shaughnessy, M., Michael, K.M., Sorkin, J.D., \& Macko, M.D. (2005). Steps after stroke: Capturing ambulatory recovery. Stroke, 36(6), 1305-7. doi: 10.1161/01.STR.0000166202.00669.d2.

Skarin, M., Sjöholm, A., Nilsson, Å.L., Nilsson, M., Bernhardt, J. \& Linden, T. (2014). A mapping study on physical activity in stroke rehabilitation: Establishing the baseline. Journal of Rehabilitation Medicine, 45(10), 9971003. doi: http://dx.doi.org/10.2340/16501977-1214.

Steffen, T.M., Hacker, T.A., \& Mollinger, L. (2002). Age and gender-related test performance in community-dwelling elderly people: Six-Minute Walk Test, Berg Balance Scale, Timed Up \& Go Test, and gait speeds. Physical Therapy, 82(2), 128-37.

Storti, K.L., Pettee, K.K., Brach, J.S., Talkowski, J.B., Richardson, C., \& Kriska A. (2008). Gait speed and step-count monitor accuracy in communitydwelling older adults. Medicine and Science in Sports and Exercise, 40(1), 59-64. doi: 10.1249/mss.0b013e318158b504

Tobias, M., Cheung, J., Carter, K., Anderson, C., \& Feigin, V. (2007). Stroke surveillance: Population-based estimates and projections for New Zealand. Australian and New Zealand Journal of Public Health, 31(6), 520-5. doi: 10.1111/j.1753-6405.2007.00136.x.

Tudor-Locke, C., Craig, C., Aoyagi, Y., Bell, R.C., Croteau, K.A., Bourdeudhuij, I., et al. (2011). How many steps/day are enough? For older adults and special populations. International Journal of Behavioural Nutrition and Physical Activity, 8(1), 80. doi:10.1186/1479-5868-8-80.

Tudor-Locke, C. (2002). Taking steps toward increased physical activity: Using pedometers to measure and motivate. President's Council on Physical Fitness and Sports Research, 3(17), 3-10.

van de Port, I.G., Kwakkel, G.,\& Lindeman, E. (2008). Community ambulation in patients with chronic stroke: How is it related to gait speed? Journal of Rehabilitation Medicine, 40(1), 23-7. doi: http://dx.doi. org/10.2340/16501977-0114. 
Van Peppen, R.P., Kwakkel, G., Wood-Dauphinee, S., Hendricks, H.J.,

Van der Wees, P.J., \& Dekker, J. (2004). The impact of physical therapy on functional outcomes after stroke: What's the evidence? Clinical Rehabilitation, 18(8), 833-862. doi: 10.1191/0269215504cr8430a.

West, T. \& Bernhardt, J. (2012). Physical activity in hospitalised stroke patients. Stroke Research and Treatment. 2012; Article ID 813765, 13 pages. doi:10.1155/2012/813765.

Wevers, L.E., Kwakkel, G., \& van de Port, I.G. (2011). Is outdoor use of the six-minute walk test with a global positioning system in stroke patients' own neighbourhoods reproducible and valid? Journal of Rehabilitation Medicine, 43(11), 1027-31. doi: http://dx.doi.org/10.2340/165019770881.

Wolf, S.L., Catlin, P.A., Gage, K., Gurucharri, K., Robertson, R. \& Stephen, K. (1999). Establishing the reliability and validity of measurements of walking time using the Emory Functional Ambulation Profile. Physical Therapy, 79(12), 1122-1133. 